\title{
Non-contact surface grating coupling for arrays of atom-photonic devices
}

\author{
A. J. VAN LANGE, ${ }^{1, *}$ (D) A. LOF, ${ }^{2}$ AND D. VAN OOSTEN ${ }^{1}$ \\ ${ }^{1}$ Debye Institute for Nanomaterials Science and Center for Extreme Matter and Emergent Phenomena, Utrecht University, Princetonplein 1, 3584 \\ CC Utrecht, The Netherlands \\ ${ }^{2}$ Center for Nanophotonics, AMOLF, Science Park 104, 1098 XG Amsterdam, The Netherlands \\ *Corresponding author: a.j.vanlange@uu.nl
}

Received 27 November 2019; revised 30 January 2020; accepted 30 January 2020; posted 31 January 2020 (Doc. ID 384110); published 3 March 2020

\begin{abstract}
For future atom-photonic devices, the incoupling method is expected to become the main bottleneck in the scaling of the number of devices, as current devices use side-coupling, which is restricted to the sample edges. This paper describes how light from above is coupled directly from free space into a freestanding membrane of $220 \mathrm{~nm}$ thin silicon nitrite $\left(\mathrm{Si}_{3} \mathrm{~N}_{4}\right)$ with surface grating couplers. The design for these couplers is modeled, captured in an elegant design formula, and experimentally tested. A coupling efficiency of $12 \%$ makes experimental coupling to an array of atom-photonic devices possible. The elegant design formula makes grating design straightforward for all-dielectric materials and wavelengths. For this formula, determination of the correct effective index of the specific waveguide mode inside the grating layer is essential. (C)2020 Optical Society of America
\end{abstract}

\section{INTRODUCTION}

In recent years, many advancements have been made in atomlight interaction in the strong coupling regime [1]. In cavity quantum electrodynamics, this strong coupling shows the promise of applications in quantum information processing [2]. Atoms have been coupled to an optical trapping field in several ways. In pioneering experiments, an ultracold atom was coupled to a microcavity with conventional mirrors [3]. The size of the cavity and consequently of the complete device has since then become smaller and smaller. As examples of such atom-nanophotonic systems, ultracold atoms were trapped in the evanescent field of light in a bottleneck resonator of a stretched fiber [4-6] and in the evanescent field of a nanocavity resonance of a $1 \mathrm{D}$ photonic crystal waveguide attached to the tip of a tapered fiber $[7,8]$. In an alternative approach, a fiber was butt-coupled to a waveguide bridge in vacuum. In its center, an alligator resonator trap was fabricated to trap the atom in its mouth [9-11]. In all these experiments, two quantum objects are strongly coupled: the photon in the nanocavity and the atom. This coupling of two dissimilar quantum systems shows great promise for quantum information processing, and these systems have shown basic device functionality in experiments. The next step is to make these experimental prototypes into easily addressable building blocks for large-scale devices.

The scaling challenge to make an array of individually addressable blocks of these devices is similar to the scaling in electrical engineering: from a single transistor to the vast arrays of transistors constituting our modern day computer chips. Bottleneck in this progress is the available area for input and output connections. Early integrated circuits had connectors around the edges creating a $1 \mathrm{D}$ contact interface. The big leap forward came from addressing the devices from the top, unlocking a $2 \mathrm{D}$ contact interface. A similar approach for atomphotonic devices demands coupling in from the top instead of butt-coupling at the edge, while preserving pristine space in the vacuum for the trapped atoms. As a result, the coupling must be through free space and at a distance. In the experiments mentioned above, the devices are addressed by a single fiber or a line of fibers from the side of the sample. Surface grating couplers offer an alternative, scalable technique to inject light directly into the device membrane layer from the top at a distance.

Grating couplers were developed in the photonics industry and have become standard practice for on-chip testing of its constituent individual devices. Typically, surface grating couplers are addressed from the top and designed to operate at small angles of incidence. The large tolerance in acceptance angle, beam size, and beam curvature make them convenient to use [12-15]. Although generally addressed by a fiber in contact with the surface, the technique is also applicable to incoming beams of light. Surface grating couplers can couple light into the device membrane directly from free space at near normal incidence angle. Therefore, a whole array of devices can be addressed by redirecting the beam from outside the vacuum chamber. The benign tolerance in acceptance angle, beam size, and beam 
curvature makes them convenient for large-scale use. For onwafer purposes, the efficiency is optimized for buried oxide layer thickness on the substrate and even an oxide overlayer $[16,17]$. For freestanding membranes of our interest, the optimum depends more heavily on other parameters, which we capture in theory and numerical simulation. Since this technique is readily applicable to any membrane, it can provide new opportunities for experiments coupling atoms to vibrating membranes [18]. The technique is also highly compatible with use in quantum gas microscopes [19-21].

In this work, we derive and numerically test an elegant design formula for grating pitch and depth. In the design, the correct determination of the effective index of the TE and TM fundamental modes plays a defining role. We follow the design to fabricate the grating couplers and show their behavior experimentally in a Fourier microscope setup.

\section{THEORY OF THE DESIGN FORMULA}

In our design, the surface grating couplers aim to couple the first grating diffraction order into the slab waveguide mode, exploiting Wood's anomaly [22]. This requires phase matching between the beam incident on the grating grooves and the guided mode in the grating layer. In a schematic representation of the process in Fig. 1, we see the beam with wave vector $\mathbf{k}$ impinging on the grating with grating pitch $\Lambda$ under an angle $\vartheta$. The supported waveguide mode has propagation constant $\beta$. The phase-matching condition for the first diffraction order is

$$
k_{z}=\beta-K,
$$

where $K=\frac{2 \pi}{\Lambda}$ and $k_{z}=\|\mathbf{k}\| \sin \vartheta$. In this equation, we see that the reciprocal lattice constant $K$ overcomes the momentum mismatch between the incoming beam and the guided mode in the grating layer. The equation can be rewritten in terms of the vacuum wavelength $\lambda_{0}$, such that $k_{z}=|| \mathbf{k}|| \sin \vartheta=\frac{2 \pi}{\lambda_{0}} \sin \vartheta$ and $\beta=\frac{2 \pi}{\lambda_{0}} n_{\text {eff, }}$, where $n_{\text {eff }}$ is the effective refractive index of the mode propagating in the grating slab. The phase-matching condition becomes

$$
\sin \vartheta=n_{\mathrm{eff}}-\frac{\lambda_{0}}{\Lambda}
$$

which leads to the following design condition for the grating pitch:

$$
\Lambda=\frac{\lambda_{0}}{n_{\text {eff }}-\sin \theta} .
$$

From these equations, the pivotal role of $n_{\text {eff }}$ in the design is apparent. In the thin device membrane grating layers of interest, the effective index depends significantly on the depth and duty cycle of the grating and on the mode profile. Because even the fundamental mode has evanescent tails extending far into the vacuum, the effective index differs significantly from the bulk index and should be calculated with care.

\section{A. Effective Index}

In the following, we consider as an example a grating in a $220 \mathrm{~nm}$ thick $\mathrm{Si}_{3} \mathrm{~N}_{4}$ layer. For vacuum wavelengths around $780 \mathrm{~nm}$, this layer supports only the fundamental transverse magnetic (TM) and transverse electric (TE) modes. The index $n_{\text {eff }}$ appearing in Eq. (3) is the effective index of these modes in the grating layer. The propagation in such a layer is in general time consuming to calculate, but simple to estimate using effective medium theory to find the bulk grating layer index and subsequent solving of Maxwell's equations for the index of the guided fundamental modes. This approach is schematically depicted in Fig. 1(b).

The refractive indices for the bulk grating layer are calculated by averaging the geometrical properties of the grating layer: thickness $a$, groove depth $d$, and duty cycle $f=w / \Lambda$, where $w$ is the grating ridge width. The particular type of averaging is determined by the orientation of the polarization with respect to the interfaces. As we will show, the zeroth-order approximation of effective medium theory [23,24] leads to excellent correspondence experiments and numerical simulations. It states that when the electric field is parallel to the interface, $n_{\text {eff }}^{2}=f n_{1}^{2}+(1-f) n_{2}^{2}$, and when it is perpendicular to the interface, $n_{\text {eff }}^{-2}=f n_{1}^{-2}+(1-f) n_{2}^{-2}$. It is important to note that the TE and TM modes in this work are defined with respect to the slab waveguide interface rather than the grating line interfaces.

We consider two averaging steps; first we average the grating grooves with the vacuum in between to find an effective grating index and subsequently average it with the remaining $\mathrm{Si}_{3} \mathrm{~N}_{4}$ (a)

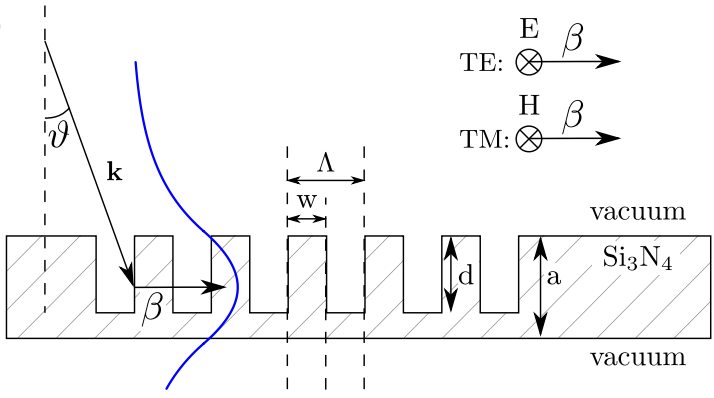

(b)

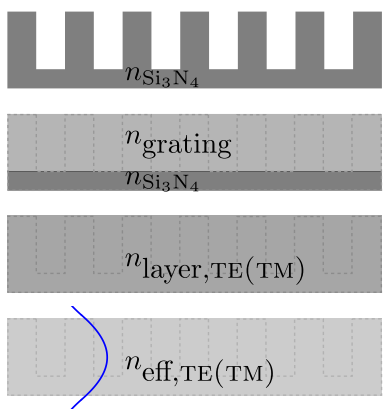

Fig. 1. (a) Schematic representation of the incoupling process and relevant design parameters. The blue line represents the vertical profile of the electric field of the TE mode. (b) Schematic representation of intermediate effective media in calculation of the effective refractive index for the fundamental TE (TM) mode in the membrane layer with grating. From top to bottom, the initial structure of the membrane, membrane after averaging the grating indices (horizontal averaging), membrane after vertical averaging, and membrane effective index for the TE (TM) mode. The blue line again represents the vertical component of the electric field of the TE mode. 
layer. The grating lines are parallel to the electric field for both the slab TE and TM modes, such that

$$
n_{\text {grating }}=\sqrt{f n_{\mathrm{Si}_{3} \mathrm{~N}_{4}}^{2}+(1-f) n_{\text {vacuum }}^{2}} .
$$

For the interface between the grating and the remaining $\mathrm{Si}_{3} \mathrm{~N}_{4}$ layer, however, the TE mode is parallel to the interface and the TM mode is perpendicular. The appropriate effective indices of the layer are thus

$$
\begin{gathered}
n_{\text {layer, } \mathrm{TE}}=\sqrt{\frac{d}{a} n_{\text {grating }}^{2}+\left(1-\frac{d}{a}\right) n_{\mathrm{Si}_{3} \mathrm{~N}_{4}}^{2},} \\
n_{\text {layer, } \mathrm{TM}}=\left(\frac{d}{a} \frac{1}{n_{\text {grating }}^{2}}+\left(1-\frac{d}{a}\right) \frac{1}{n_{\mathrm{S}_{3} \mathrm{~N}_{4}}^{2}}\right)^{-\frac{1}{2}} .
\end{gathered}
$$

Subsequently, Maxwell's equations are solved for a layered medium of a slab with index $n_{\text {layer,TE(TM) }}$ surrounded by vacuum, with the appropriate boundary conditions for TE (TM) mode. The derivation follows the lines of Burke [25], but we solve the equation numerically rather than graphically. As an example, for $d=\frac{3}{4} a$ and $f=\frac{1}{2}$, which are the values for optimum coupling for similar grating structures $[16,26]$, we find values $n_{\text {grating }}=1.58, n_{\text {layer, } \mathrm{TE}}=1.69$, and $n_{\text {layer, } \mathrm{TM}}=1.65$. The resulting $n_{\text {eff }}$ for the fundamental TE and TM modes are wavelength dependent, and the values for $\lambda_{0} \simeq 780 \mathrm{~nm}$ are

$$
n_{\mathrm{eff}, \mathrm{TE}} \simeq 1.41 \text { and } \quad n_{\mathrm{eff}, \mathrm{TM}} \simeq 1.20 .
$$

Note that these indices differ considerably from the bulk indices $n_{\text {layer,TE }}$ and $n_{\text {layer,TM }}$ due to the spatial extent of the mode wings into the vacuum. Moreover, these indices are significantly different from those of the modes supported by a $220 \mathrm{~nm}$ slab of $\mathrm{Si}_{3} \mathrm{~N}_{4}$ without a grating, which are $n_{\text {slab }, \mathrm{TE}}=1.71$ and $n_{\text {slab }, \mathrm{TM}}=1.41$ for $\lambda_{0}=780 \mathrm{~nm}$.

\section{NUMERICAL CALCULATIONS}

To test the design condition described above, the Stanford Stratified Structure Solver $\left(S^{4}\right)$ Python package is used $\left(S^{4}\right.$ version 1.1 downloaded from https://web.stanford.edu/group/fan/S4/install.html). $S^{4}$ is a frequency domain code that uses rigorous coupled wave analysis and an S-matrix algorithm [27] and is suitable to calculate transmission through a patterned layer, i.e., a grating. Convergence is verified for the used Fourier basis of 50 Fourier orders. The excitation plane wave is varied in incident angle, frequency, and polarization. The forward transmission is measured under the grating layer over the entire grating layer. As the simulation is a closed system, the rest of the light is necessarily scattered in the backward direction. We exploit Wood's anomaly to attribute a sharp decrease in the transmission to the opening of a new reflection channel, namely, light coupled into the grating layer and coupled out in the backward direction. For a plane wave, this process is so effective that the transmission becomes zero due to the destructive interference between different transmission paths.

Figure 2 shows the backward scattered fraction for a $220 \mathrm{~nm}$ thick slab with $d=170 \mathrm{~nm}$ and $\Lambda=643 \mathrm{~nm}$ for $s$ and $p$ polarizations. The maxima in these plots occur on specific lines in angle-wavelength space. They are symmetric around zero angle, because there is no preference in direction of the excited mode. For $s(p)$ polarization, the lines along which maxima occur clearly match with the calculated diffraction condition curves for the TE (TM) mode in this slab, which are indicated by the dashed lines. The design formula has thus proved to be effective and the determination of $n_{\text {eff }}$ accurate. The TE (TM) mode is expected to be excited by $s(p)$ polarization, because the electric fields of the incoming beam and the slab waveguide mode are oriented in the same direction.

Our primary interest is in the TM mode in the grating layer, because this mode has the largest evanescent tails above the waveguide and is thus most suitable for atom-photonic applications. In experiment, we therefore investigate $p$-polarized light coupling to the TM mode, for which we first design and fabricate the grating couplers. (a) s polarization

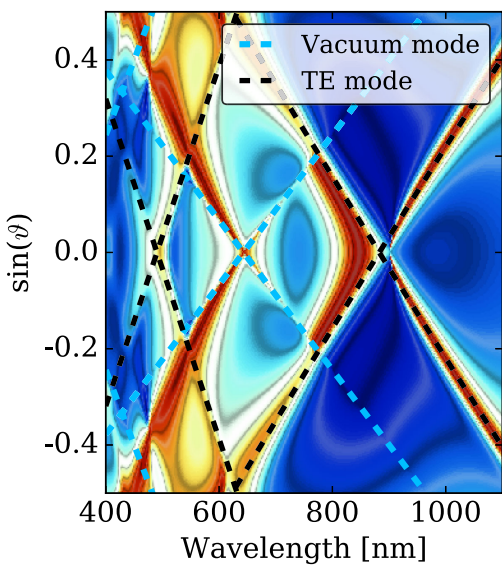

(b) p polarization

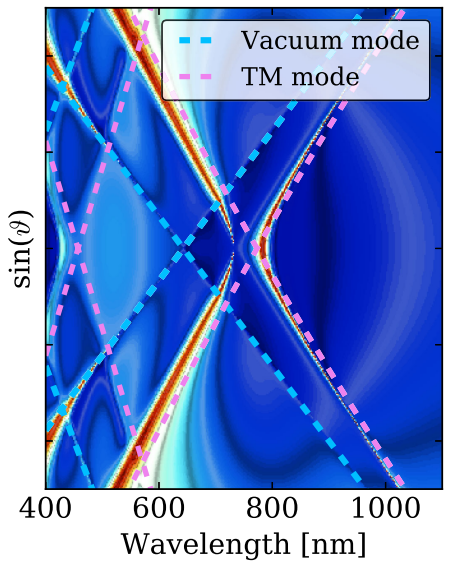

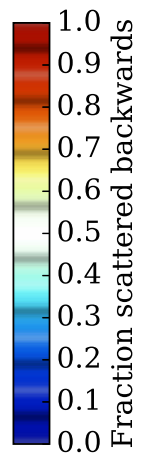

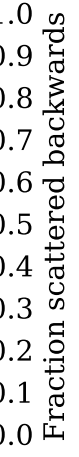

Fig. 2. Color plot of backward flux through a line just above a grating layer. The dashed lines are solutions of the diffraction equation [Eq. (2)] for the TE mode with $n_{\text {eff }}=n_{\text {eff,TE}}$, the TM mode with $n_{\text {eff }}=n_{\text {eff,TM }}$, and the vacuum mode with $n_{\text {eff }}=1$. 

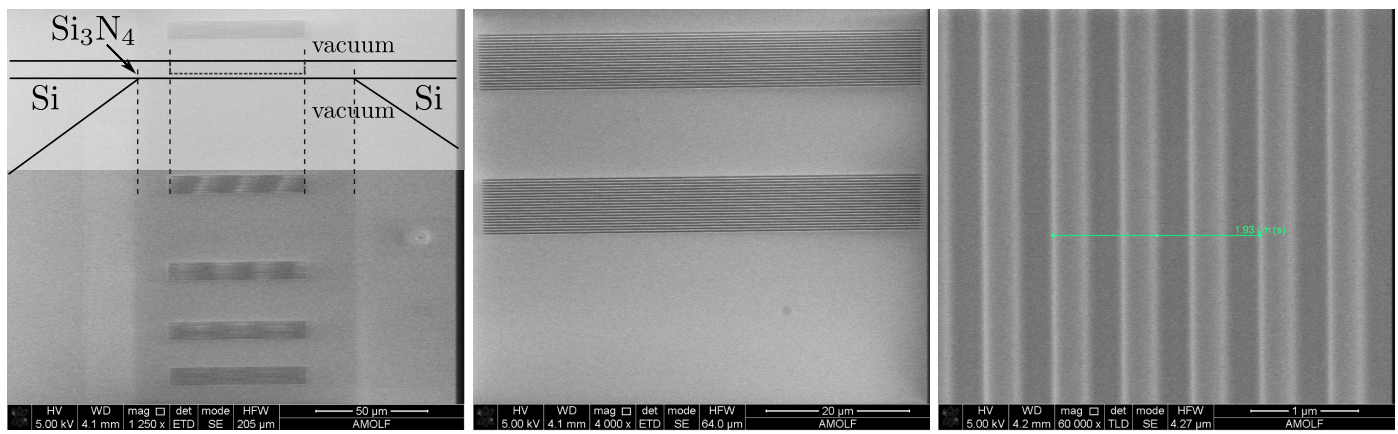

Fig. 3. Cross-section view schematic (top left overlay) and top view $S E M$ pictures of a $\mathrm{Si}_{3} \mathrm{~N}_{4}$-membrane with five fabricated grating couplers. Grating couplers consist of 20 lines with pitch $643 \mathrm{~nm}$ and duty cycle of 0.5 . The spacings between the gratings are $20,30,50$, and $100 \mu \mathrm{m}$.

\section{GRATING FABRICATION}

The fabrication process starts with an array of membranes of stoichiometric $\mathrm{Si}_{3} \mathrm{~N}_{4}(n=2.014)$ purchased from Small Tech Supplies. The free-standing membranes are on a silicon support and have dimensions of $1 \mathrm{~mm} \times 100 \mu \mathrm{m}$ and $220 \mathrm{~nm}$ thickness.

The surface grating couplers are fabricated using focused ion beam (FIB) milling at the nanocenter of NWO institute AMOLF in Amsterdam [FEI Helios dual beam FIB (Ga+ milling) set to $30 \mathrm{kV}$ acceleration voltage, $280 \mathrm{pA}$ ion beam current, $26 \mu$ s dwell time and 30 loops]. Grooves are milled to a depth of $\frac{3}{4}$ of the thickness of the slab and with a duty cycle of $\frac{1}{2}$, because these values have proven to give the highest coupling efficiency for similar structures [16,26]. The pitch is varied between 640 and $700 \mathrm{~nm}$. On each of the membranes, one pitch is chosen for the gratings on that membrane. Gratings of 20 lines of $60 \mu \mathrm{m}$ wide are placed $20,30,50$, and $100 \mu \mathrm{m}$ apart to test the transmission. Figure 3 shows electron microscope images and a cross-section schematic of one of the resulting membranes.

\section{EXPERIMENTAL SETUP}

Samples are characterized in a microscopy setup reported in Ref. [28] with the addition of an acquisition arm (see Fig. 4). The setup combines real space and Fourier space control. More particularly, the setup can be used for controlling the incidence angle of light that is offered for incoupling at one location in the field of view of a microscope objective, while at the same time giving access to real-space imaging of outcoupling of light at another position in the field of view. Ref. [28] provides a detailed and accurate description of the setup components as used in this work. The setup is shown schematically in Fig. 4.

The sample is mounted on a $x, y, z$-translation stage and positioned in the focus of the microscope objective. The excitation beam originating from a supercontinuum white light laser arrives on the table through a paddle polarization controller. The beam undergoes polarization and spatial filtering before arriving at two galvo mirrors on the bottom right. The two galvo mirrors allow the beam angle to be scanned in two directions. The telescope after the galvo mirrors, together with the Fourier lens, translates this angle to a well-defined spot on the back focal plane (BFP), thereby selecting a specific angle of excitation of

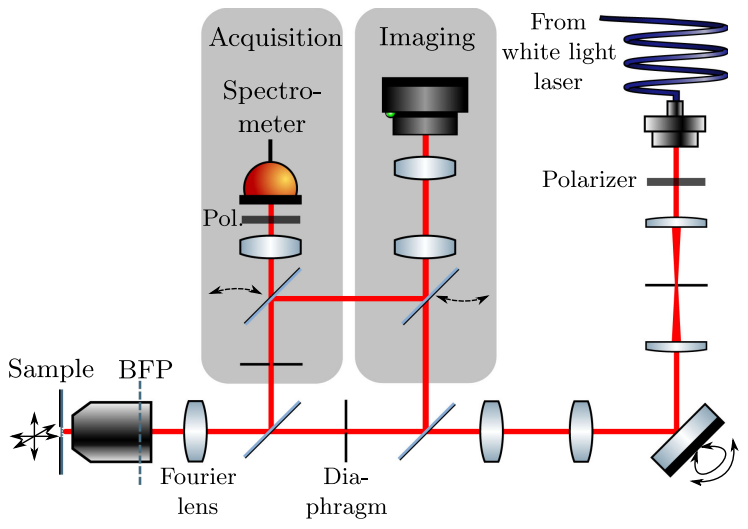

Fig. 4. Fourier microscope setup at NWO institute AMOLF used to characterize the grating samples. The light source is a supercontinuum white light laser (NKT photonics SuperK extreme), the microscope objective is an Olympus 100x, NA 0.95 M Plan IR objective, and the acquisition is performed by an Avantes peltier cooled Si CCD array spectrometer (AvaSpec-2048TEC-USB2-2).

the sample. The spot is scanned over the BFP of the objective to excite over a large range of angles consecutively.

In addition to the angle, we can also select a specific location for excitation on the sample. In the intermediate image plane before the Fourier lens, a movable diaphragm ensures that only the incoupling grating is illuminated. The sample (as well as the diaphragm opening) is imaged on the camera through the imaging arm. The sample can also be imaged through the acquisition arm by turning in a flip mirror. In this arm, a second diaphragm is placed to select the outcoupling grating. With the flip mirror in the acquisition arm removed, the light is sent to a spectrometer. This way, we scan the angle of the excitation and measure the spectrum from the outcoupling grating. A polarization filter can be placed in the acquisition arm for cross and parallel polarization measurements.

\section{RESULTS}

In the experiment, light is coupled through a $220 \mathrm{~nm}$ thick $\mathrm{Si}_{3} \mathrm{~N}_{4}$ membrane in air by two surface grating couplers separated by $20 \mu \mathrm{m}$. Figure 5 shows the wavelength-angle dependence of transmission of $p$-polarized excitation light with grating couplers with a pitch of $643 \mathrm{~nm}$. The outcoupled light is 


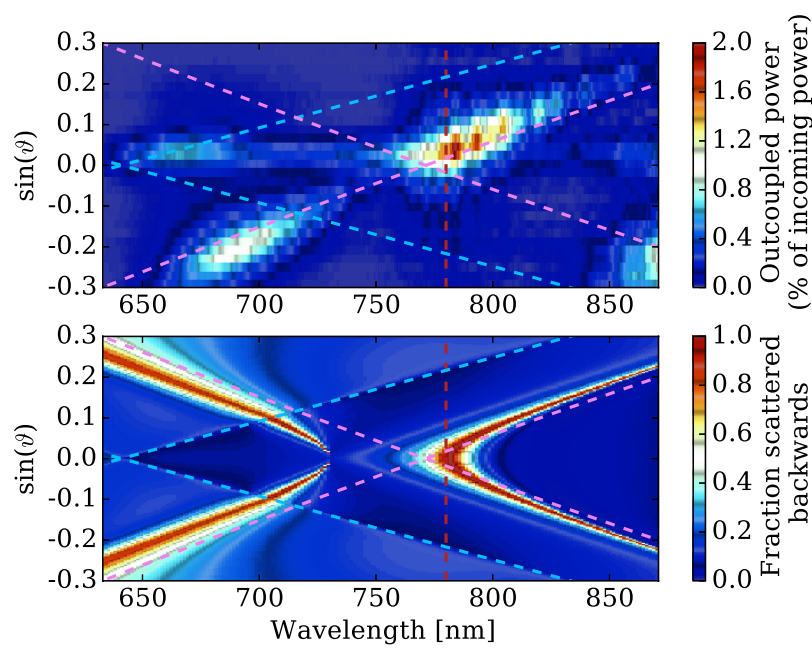

Fig. 5. Experimental results (top) and simulation (bottom) of transmission of $p$-polarized light through a $220 \mathrm{~nm}$ thin slab of $\mathrm{Si}_{3} \mathrm{~N}_{4}$ surrounded by air. In- and outcoupling through a surface grating coupler with pitch $643 \mathrm{~nm}$, groove depth $170 \mathrm{~nm}$. Dashed lines are grating conditions for the TM mode (pink) and air mode (blue) and the resonance wavelength of rubidium (red) at $780 \mathrm{~nm}$.

verified to have parallel polarization. The results are compared to the simulation for the same grating parameters. The diffraction conditions from Eq. (2) are plotted for air $\left(n_{\text {eff }}=1\right)$ and the TM mode in the grating slab $\left(n_{\text {eff }}=n_{\text {eff,TM }}\right)$.

The maxima in transmission appear on the diffraction condition for the TM mode and also coincide with the maxima of the simulation. This further confirms the effectiveness of the design formula and the accuracy of the $n_{\text {eff }}$ determination. In comparison with the simulation results, we note the following differences.

First, the experiment shows no signal for opposite angles, whereas the simulation does. The simulation considers an infinite grating layer and captures modes excited in both positive and negative directions. In the experiment, only the modes directed towards the outcoupling grating can be measured. Indeed, if the measured transmission direction is inverted by switching the selected in- and outcoupling grating, the graph is mirrored in the line of $0 \hat{\mathrm{A}}^{\circ}$. Second, in the experimental data, the high transmission region near the diffraction condition line has a substructure with several maxima separated by $\sim 10 \mathrm{~nm}$. We attribute these maxima to interference caused by partial reflection on the interfaces between the grating layer and the regular $\mathrm{Si}_{3} \mathrm{~N}_{4}$ waveguide layer, which is not included in the simulation. In particular, the $20 \mu \mathrm{m}$ long $\mathrm{Si}_{3} \mathrm{~N}_{4}$ waveguide slab between the gratings would give rise to etalon modes with roughly the same wavelength separation of $\sim 10.5 \mathrm{~nm}$.

To determine the transmission efficiency, the outcoupled power is compared to incident power, which is measured by the spectrometer just before entering the microscope objective. Importantly, we see there is considerable transmission at $780 \mathrm{~nm}$ at near normal incidence. This shows that the design works, and we understand how the incoupling depends on the design parameters.

To determine the optimum pitch for a given wavelength, we measure transmission for gratings with varying pitches on

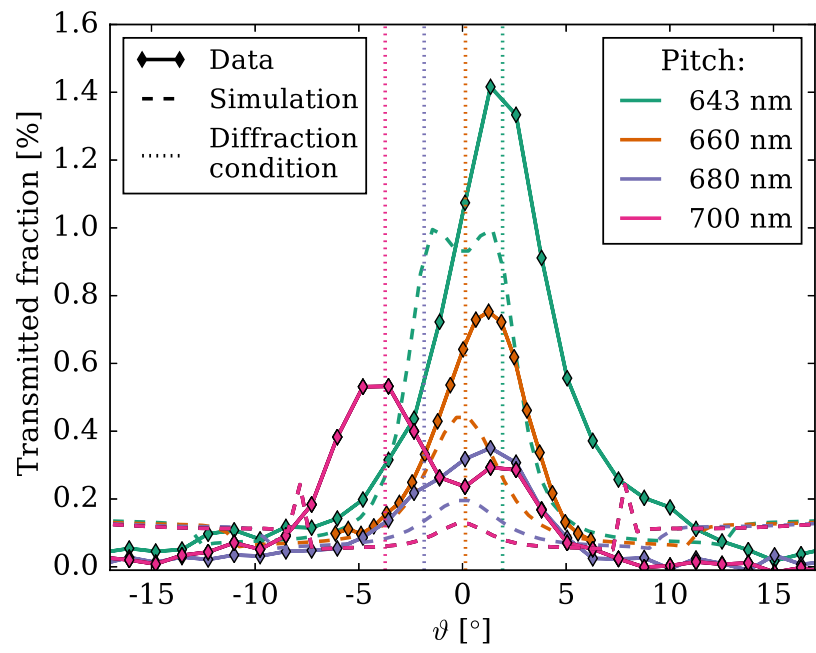

Fig. 6. Transmission of $780 \mathrm{~nm}$ light for several pitches. Each pitch has its own color. For each pitch, the diffraction condition from Eq. (2) for $n_{\text {eff }}$ of the grating layer (dashed-dotted vertical line), the results from simulation (dashed line), and data from experiments (connected diamonds) are drawn.

several membranes. We take the crosscuts at $780 \mathrm{~nm}$ as shown in Fig. 5. For all the pitches, we combine the experimental data, simulation output, and diffraction condition in Fig. 6.

Figure 6 shows that design, simulation, and experiment are in good qualitative agreement. The shifts in the transmission maximum for different pitches exhibit the same trend. Therefore, the design condition of Eq. (3) properly describes the position of maximum transmission. The most suitable pitch out of this collection is $643 \mathrm{~nm}$ with a maximum transmission of almost $1.4 \%$ at near normal incidence. The total transmission of $1.4 \%$ indicates that the single coupler efficiency is $12 \%$. This value is a lower limit, because the losses in the remainder of the waveguide and in the setup are included in the efficiency. The efficiency compares well to the values found in literature for a $\mathrm{Si}_{3} \mathrm{~N}_{4}$ grating coupler with an unoptimized buried oxide layer and without an overgrowth layer [16] and for a $\mathrm{Si}_{3} \mathrm{~N}_{4}$ grating coupler suspended in $\mathrm{SiO}_{2}$ [12]. More advanced structures of grating couplers in suspended waveguides of $\mathrm{Si}$ and $\mathrm{Ge}$ show great increase in efficiency and functionality, which may also provide good prospects for use in silicon nitride [29,30].

\section{CONCLUSION}

In conclusion, we find an elegantly simple design condition for efficient coupling and transmission at any desired wavelength and angle in thin dielectric waveguide layers. The design condition is confirmed by simulation results for a single grating coupler. We used the condition to design and fabricate surface grating couplers with efficiency as high as $12 \%$, and achieved a total transmission efficiency through the incoupler-waveguideoutcoupler system of $1.4 \%$. For light with a wavelength of $780 \mathrm{~nm}$, the design shows optimum light transmission through a $220 \mathrm{~nm}$ thick slab of $\mathrm{Si}_{3} \mathrm{~N}_{4}$ for a surface grating coupler with 20 grooves with a pitch of $643 \mathrm{~nm}$ at a depth of $170 \mathrm{~nm}$. For any choice of wavelength, dielectric material, and thickness of the waveguide, optimum incoupling parameters can be easily 
calculated using the design formula with the correct guided mode effective index.

Funding. Nederlandse Organisatie voor Wetenschappelijk Onderzoek.

Acknowledgment. The authors thank Marko Kamp, Hugo Doeleman, and Femius Koenderink for the access to and the support on the Fourier microscope setup, Hans Zeijlemakers for fabrication advice, and Kostas Voutyras and Peter van der Straten for lab support and fruitful discussions.

Disclosures. The authors declare that there are no conflicts of interest related to this paper.

\section{REFERENCES}

1. A. F. Kockum, A. Miranowicz, S. D. Liberato, S. Savasta, and F. Nori, "Ultrastrong coupling between light and matter," Nat. Rev. Phys. 1, 19-40 (2019).

2. L. Carroll, J.-S. Lee, C. Scarcella, K. Gradkowski, M. Duperron, H. Lu, Y. Zhao, C. Eason, P. Morrissey, M. Rensing, S. Collins, H. Y. Hwang, and P. O'Brien, "Photonic packaging: transforming silicon photonic integrated circuits into photonic devices," Appl. Sci. 6, 426 (2016).

3. G. Rempe, R. J. Thompson, R. J. Brecha, W. D. Lee, and H. J. Kimble, "Optical bistability and photon statistics in cavity quantum electrodynamics," Phys. Rev. Lett. 67, 1727-1730 (1991).

4. M. Scheucher, A. Hilico, E. Will, J. Volz, and A. Rauschenbeutel, "Quantum optical circulator controlled by a single chirally coupled atom," Science 354, 1577-1580 (2016).

5. A. Dareau, Y. Meng, P. Schneeweiss, and A. Rauschenbeutel, "Observation of ultrastrong spin-motion coupling for cold atoms in optical microtraps," Phys. Rev. Lett. 121, 253603 (2018).

6. Y. Meng, A. Dareau, P. Schneeweiss, and A. Rauschenbeutel, "Nearground-state cooling of atoms optically trapped $300 \mathrm{~nm}$ away from a hot surface," Phys. Rev. X 8, 031054 (2018).

7. T. G. Tiecke, K. P. Nayak, J. D. Thompson, T. Peyronel, N. P. de Leon, V. Vuletić, and M. D. Lukin, "Efficient fiber-optical interface for nanophotonic devices," Optica 2, 70-75 (2015).

8. J. D. Thompson, T. G. Tiecke, N. P. de Leon, J. Feist, A. V. Akimov, M. Gullans, A. S. Zibrov, V. Vuletić, and M. D. Lukin, "Coupling a single trapped atom to a nanoscale optical cavity," Science 340, 1202-1205 (2013).

9. A. Goban, C.-L. Hung, S.-P. Yu, J. D. Hood, J. A. Muniz, J. H. Lee, M. J. Martin, A. C. McClung, K. S. Choi, D. E. Chang, O. Painter, and H. J. Kimble, "Atom-light interactions in photonic crystals," Nat. Commun. 5, 3808 (2014).

10. C.-L. Hung, S. M. Meenehan, D. E. Chang, O. Painter, and H. J. Kimble, "Trapped atoms in one-dimensional photonic crystals," New J. Phys. 15, 083026 (2013).

11. J. S. Douglas, H. Habibian, C.-L. Hung, A. V. Gorshkov, H. J. Kimble, and D. E. Chang, "Quantum many-body models with cold atoms coupled to photonic crystals," Nat. Photonics 9, 326-331 (2015).

12. C. R. Doerr, L. Chen, Y.-K. Chen, and L. L. Buhl, "Wide bandwidth silicon nitride grating coupler," IEEE Photon. Technol. Lett. 22, 1461-1463 (2010).
13. A. Z. Subramanian, S. Selvaraja, P. Verheyen, A. Dhakal, K. Komorowska, and R. Baets, "Near-infrared grating couplers for silicon nitride photonic wires," IEEE Photon. Technol. Lett. 24, 1700-1703 (2012).

14. C. Lacava, S. Stankovic, A. Z. Khokhar, T. D. Bucio, F. Y. Gardes, G. T. Reed, D. J. Richardson, and P. Petropoulos, "Si-rich silicon nitride for nonlinear signal processing applications," Sci. Rep. 7, 22 (2017).

15. J. Hong, A. M. Spring, F. Quu, and S. Yokoyama, "A high efficiency silicon nitride waveguide grating coupler with a multilayer bottom reflector," Sci. Rep. 9, 1 (2019).

16. G. Maire, L. Vivien, G. Sattler, A. Kazmierczak, B. Sanchez, K. B. Gylfason, A. Griol, D. Marris-Morini, E. Cassan, and D. Giannone, "High efficiency silicon nitride surface grating couplers," Opt. Express 16, 328-333 (2008)

17. H. Zhang, C. Li, X. Tu, J. Song, H. Zhou, X. Luo, Y. Huang, M. Yu, and G. Q. Lo, "Efficient silicon nitride grating coupler with distributed Bragg reflectors," Opt. Express 22, 21800-21805 (2014).

18. A. Vochezer, T. Kampschulte, K. Hammerer, and P. Treutlein, "Lightmediated collective atomic motion in an optical lattice coupled to a membrane," Phys. Rev. Lett. 120, 073602 (2018).

19. W. S. Bakr, J. I. Gillen, A. Peng, S. Fölling, and M. Greiner, "A quantum gas microscope for detecting single atoms in a Hubbard-regime optical lattice," Nature 462, 74-77 (2009).

20. L. W. Cheuk, M. A. Nichols, M. Okan, T. Gersdorf, V. V. Ramasesh, W. S. Bakr, T. Lompe, and M. W. Zwierlein, "Quantum-gas microscope for fermionic atoms," Phys. Rev. Lett. 114, 193001 (2015).

21. A. Omran, M. Boll, T. A. Hilker, K. Kleinlein, G. Salomon, I. Bloch, and C. Gross, "Microscopic observation of Pauli blocking in degenerate fermionic lattice gases," Phys. Rev. Lett. 115, 263001 (2015).

22. R. W. Wood, "On a remarkable case of uneven distribution of light in a diffraction grating spectrum," Proc. Phys. Soc. London 18, 269-275 (1901).

23. S. Rytov, "Electromagnetic properties of a finely stratified medium," Sov. Phys. JETP 2, 466-475 (1956).

24. R. Halir, P. J. Bock, P. Cheben, A. Ortega-Moñux, C. Alonso-Ramos, J. H. Schmid, J. Lapointe, D.-X. Xu, J. G. Wangüemert-Pérez, I. Molina-Fernández, and S. Janz, "Waveguide sub-wavelength structures: a review of principles and applications," Laser Photon. Rev. 9, 25-49 (2015).

25. J. J. Burke, "Propagation constants of resonant waves on homogeneous, isotropic slab waveguides," Appl. Opt. 9, 2444-2452 (1970).

26. D. Vermeulen, S. Selvaraja, P. Verheyen, G. Lepage, W. Bogaerts, P. Absil, D. Van Thourhout, and G. Roelkens, "High-efficiency fiber-tochip grating couplers realized using an advanced CMOS-compatible silicon-on-insulator platform," Opt. Express 18, 18278-18283 (2010).

27. V. Liu and S. Fan, "S4: a free electromagnetic solver for layered periodic structures," Comput. Phys. Commun. 183, 2233-2244 (2012).

28. F. Bernal Arango, A. Kwadrin, and A. F. Koenderink, "Plasmonic antennas hybridized with dielectric waveguides," ACS Nano 6, 10156-10167 (2012).

29. Z. Cheng and H. K. Tsang, "Experimental demonstration of polarization-insensitive air-cladding grating couplers for silicon-on-insulator waveguides," Opt. Lett. 39, 2206-2209 (2014).

30. J. Kang, Z. Cheng, W. Zhou, T.-H. Xiao, K.-L. Gopalakrisna, M. Takenaka, H. K. Tsang, and K. Goda, "Focusing subwavelength grating coupler for mid-infrared suspended membrane germanium waveguides," Opt. Lett. 42, 2094-2097 (2017). 